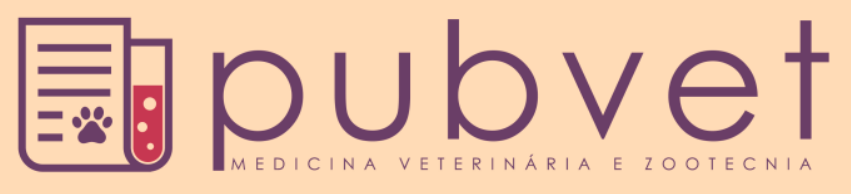

https://doi.org/10.22256/pubvet.v12n4a68.1-7

\title{
Rendimento de forragem, composição química e morfogênese de Megathyrsus maximus cv. Tanzânia-1 sob frequências de desfolhação
}

\author{
Newton de Lucena Costa ${ }^{\ominus}$, Liana Jank ${ }^{\ominus 2}$, Fabíola Helena dos Santos Fogaça ${ }^{\bullet}$, João \\ Avelar Magalhães 4, Amaury Burlamaqui Bendahan ${ }^{0}$, Francisco José de Seixas \\ Santos ${ }^{0}$, Braz Henrique Nunes Rodrigues $\bullet 6$
}

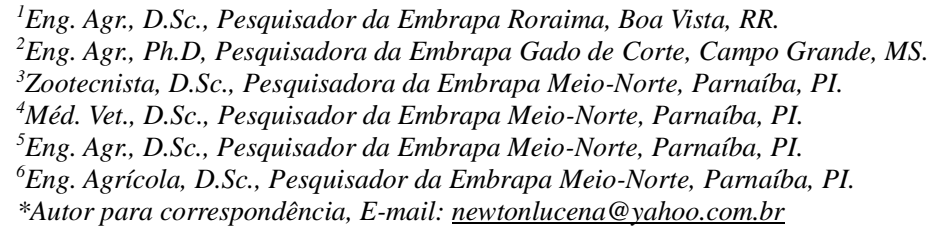

RESUMO. O efeito da frequência de desfolhação (14, 21, 28, 35 e 42 dias) sobre o rendimento e composição química da forragem e características morfogênicas e estruturais de Megathyrsus maximus cv. Tanzânia-1 foi avaliado em condições de campo. O aumento da frequência de desfolhação resultou em maiores rendimentos de matéria seca verde (MSV), contudo, implicou em decréscimos significativos dos teores de nitrogênio, fósforo, cálcio, magnésio e potássio. As taxas de aparecimento e a densidade populacional de perfilhos são inversamente proporcionais aos períodos de descanso, ocorrendo o inverso quanto a taxa de senescência foliar. Os maiores rendimentos de MSV, número de folhas vivas perfilho ${ }^{-1}$, comprimento médio de folhas, taxa de expansão foliar e índice de área foliar foram obtidos com frequências de desfolhação de 38,5; 32,3; 40,2; 24,1 e 34,7 dias, respectivamente. A frequência de desfolhação mais adequada para pastagens de $M$. maximus cv. Tanzânia-1, visando a conciliar produção, vigor de rebrota e qualidade da forragem, situa-se entre 35 e 40 dias.

Palavras chave: composição química, folhas, matéria seca, perfilhamento, senescência

\section{Forage yield, chemical composition and morphogenesis of Megathyrsus maximus cv. Tanzânia-1 under defoliation frequencies}

\begin{abstract}
The effects of defoliation frequencies (14, 21, 28, 35 and 42 days) on green dry matter (GDM) yield, chemical composition and morphogenetic and structural characteristics of Megathyrsus maximus cv. Tanzânia-1 were evaluated under natural field conditions. The increase of defoliation frequency resulted in higher GDM yields, however implied significant decreases in the levels of nitrogen, phosphorus, calcium, magnesium and potassium. The leaf appearance rate and populational tiller density are inversely proportional to defoliation, occurring the inverse for foliar senescence rate. Maximum GDM yields, number of leaves tiller ${ }^{-1}$, average leaf length, leaf expansion rate and leaf area index were obtained with defoliation frequencies of $38.5 ; 32.3 ; 40.2 ; 24.1$ and 34.7 days, respectively. These data suggest that grass grazing at 35 to 40 days of rest period were optimal for obtain maximum yields and regrowth of rich forage.
\end{abstract}

Keywords: chemical composition, dry matter, leaves, tillering, senescence 


\title{
Rendimiento de forraje, composición química y morfogénesis de Megathyrsus maximus cv. Tanzânia-1 bajo frecuencias de defoliación
}

\begin{abstract}
RESUMEN. El efecto de frecuencias de defoliación (14, 21, 28, 35 y 42 días) en la producción, composición química del forraje y las características morfogenéticas y estructurales de Megathyrsus maximus cv. Tanzânia-1 se evaluó en condiciones de campo. Aumentar la frecuencia de defoliación resultó en mayores rendimientos de materia seca verde (MSV), sin embargo, resultó en reducciones significativas de los contenidos de nitrógeno, fósforo, calcio, magnesio y potasio. Las tasas de aparición de las hojas y la densidad poblacional de macollas son inversamente proporcional a las frecuencias de defoliación, mientras que lo contrario ocurrió para la tasa de senescencia de las hojas. Los mayores rendimientos de MSV, número de hojas por macollas, longitud media de las hojas, tasa de expansión de hojas e índice de área foliar se obtuvieron a 38,5; 32,3; 40,2; 24,1 e 34,7 días, respectivamente. La frecuencia de defoliación más adecuada para el pastoreo de pasturas de M. maximus cv. Tanzânia-1, destinada a conciliar la producción, el vigor de rebrote y la calidad del forraje, está entre 35 y 40 días de descanso.
\end{abstract}

Palabras clave: composición química, hojas, macollaje, materia seca, senescencia

\section{Introdução}

Em Roraima, as pastagens cultivadas representam o mais importante recurso forrageiro para a alimentação de bovinos de corte e/ou leite. A utilização de práticas inadequadas de manejo, notadamente o pastejo contínuo ou com períodos mínimos de descanso e altas intensidades de desfolhação contribuem para baixa disponibilidade e qualidade da forragem, com reflexos negativos nos índices de desempenho zootécnico dos animais. A produtividade da pastagem é fortemente influenciada pelas condições ambientais (temperatura, luz, água e fertilidade do solo) e práticas de manejo, enquanto que sua perenidade decorre, entre outros fatores, da capacidade de reconstituição e manutenção da área foliar após a desfolhação, a qual afeta a estrutura do dossel, determinando sua velocidade de crescimento, acúmulo de forragem, composição química e persistência (Nabinger \& Pontes, 2002). A frequência de desfolhação e o período de descanso disponibilizado para o crescimento da pastagem afeta sua produtividade, composição química, capacidade de rebrota e persistência. Pastejos frequentes proporcionam maiores rendimentos de forragem, porém, concomitantemente, ocorrem decréscimos acentuados em sua composição química, com maior acúmulo de material fibroso, decréscimo na relação folha/colmo e, consequentemente, menor consumo pelos animais (Lemaire et al., 2011).

$\mathrm{O}$ manejo adequado de pastagens consiste na mediação do encontro planta-animal visando maior produtividade e qualidade da forragem em oferta, sem afetar sua persistência, além da manutenção ou melhoria da integridade das características, físicas, químicas e biológicas do solo. A partir do equilíbrio entre produção e qualidade da forragem, os requerimentos nutricionais dos animais podem ser garantidos, refletindo em melhor desempenho zootécnico dos animais. A produtividade das gramíneas forrageiras decorre da contínua emissão de folhas e perfilhos, mecanismo que assegura a restauração da área foliar após o pastejo e sua perenidade. Os processos de formação e desenvolvimento de folhas são fundamentais para o crescimento vegetal, considerando-se a importância das folhas no processo de fotossíntese, ponto de partida para a formação de novos tecidos (Cecato et al., 2000, Santos et al., 2012). A morfogênese de gramíneas forrageiras, durante seu crescimento vegetativo, caracteriza-se por três fatores: a taxa de aparecimento, a taxa de alongamento e a longevidade das folhas. A taxa de aparecimento e a longevidade das folhas determinam o número de folhas vivas/perfilho, as quais são geneticamente determinadas e podem ser afetadas pelos fatores ambientais e as práticas de manejo adotadas (Nabinger \& Pontes, 2002, Lemaire et al., 2011).

Neste trabalho foram avaliados os efeitos da frequência de desfolhação sobre a produtividade de forragem, composição química e características morfogênicas e estruturais de Megathyrsus maximus cv. Tanzânia-1 nos cerrados de Roraima.

\section{Material e Métodos}

O ensaio foi conduzido no Campo Experimental da Embrapa Roraima, localizado em 
Boa Vista, durante o período de Maio a Setembro de 2015, correspondendo uma precipitação acumulada de $1.218,9 \mathrm{~mm}$ e temperatura média mensal de $24,86^{\circ} \mathrm{C}$. O solo da área experimental é um Latossolo Amarelo, textura média, fase cerrado, com as seguintes características químicas, na profundidade de $0-20 \mathrm{~cm}$ : $\mathrm{pH}_{\mathrm{H} 2 \mathrm{O}}=4,9 ; \mathrm{P}=2,9$ $\mathrm{mg} / \mathrm{kg} ; \mathrm{Ca}+\mathrm{Mg}=1,19$ cmol $_{\mathrm{c}} \cdot \mathrm{dm}^{-3} ; \mathrm{K}=0,019$ $\mathrm{cmol}_{\mathrm{c}} \cdot \mathrm{dm}^{-3} \mathrm{e} \mathrm{Al}=0,57 \mathrm{cmol}_{\mathrm{c}} \mathrm{dm}^{-3}$.

$\mathrm{O}$ delineamento experimental foi inteiramente casualizado com três repetições. Os tratamentos consistiram de cinco frequências de desfolhação $(14,21,28,35$ e 42 dias). A adubação de estabelecimento constou da aplicação de $90 \mathrm{~kg}$ de $\mathrm{N} \mathrm{ha}^{-1}, 50 \mathrm{~kg}$ de $\mathrm{P}_{2} \mathrm{O}_{5}$ ha $^{-1}$ e $60 \mathrm{~kg} \mathrm{de}_{2} \mathrm{O}_{\text {ha }}{ }^{-1}$, sob a forma de ureia, superfosfato triplo e cloreto de potássio, respectivamente. A adubação nitrogenada foi parcelada em três vezes, sendo $1 / 3$ quando do plantio, $1 / 3$ aos 21 dias e 1/3 aos 35 dias. As parcelas mediam 2,0 x 2,0 m, sendo a área útil de $1,0 \mathrm{~m}^{2}$. Durante o período experimental foram realizados $10,7,5,4$ e 3 cortes, respectivamente para frequências de desfolhação de 14, 21, 28, 35 e 42 dias e a uma altura de $30 \mathrm{~cm}$ acima do solo.

Os parâmetros avaliados foram rendimento de matéria seca verde (MSV), teores de nitrogênio $(\mathrm{N})$, fósforo $(\mathrm{P})$, cálcio $(\mathrm{Ca})$, magnésio $(\mathrm{Mg})$ e potássio $(\mathrm{K})$, número de folhas vivas perfilho ${ }^{-1}$ (NFV), taxa de aparecimento de folhas (TAF), taxa de expansão foliar (TEF), densidade populacional de perfilhos (DPP), taxa de senescência foliar (TSF), comprimento médio de folhas (CMF) e índice de área foliar (IAF). A TEF e a TAF foram calculadas dividindo-se o comprimento acumulado de folhas e o número total de folhas no perfilho, respectivamente, pelo período de rebrota. O CMF foi determinado pela divisão do alongamento foliar total do perfilho pelo número de folhas. Para o cálculo da área foliar foram coletadas amostras de folhas verdes completamente expandidas, procurando-se obter uma área entre 200 e $300 \mathrm{~cm}^{2}$. As amostras foram digitalizadas e a área foliar estimada com o auxílio de planímetro ótico eletrônico (Li-Cor 3100C). Posteriormente, as amostras foram levadas à estufa com ar forçado a $65^{\circ} \mathrm{C}$ até atingirem peso constante, obtendo-se a MS foliar. A área foliar específica (AFE) foi determinada através da relação entre a área de folhas verdes e a sua MS $\left(\mathrm{m}^{2} / \mathrm{g}\right.$ MS foliar). O IAF foi determinado a partir do produto entre a MS total das folhas verdes ( $\mathrm{g}$ de $\left.\mathrm{MS} / \mathrm{m}^{2}\right)$ pela AFE $\left(\mathrm{m}^{2} / \mathrm{g}\right.$ de MS foliar). A TSF foi obtida dividindo-se o comprimento da folha que se apresentava de coloração amarelada ou necrosada pela idade de rebrota.

Os dados foram submetidos à análise de variância e de regressão considerando o nível de significância de $5 \%$ de probabilidade. Para se estimar a resposta dos parâmetros avaliados às frequências de desfolhação, a escolha dos modelos de regressão baseou-se na significância dos coeficientes linear e quadrático, por meio do teste "t", de Student, ao nível de 5\% de probabilidade.

\section{Resultados e Discussão}

As frequências de desfolhação afetaram $(\mathrm{P}<0,05)$ os rendimentos de MSV, sendo a relação quadrática e o máximo valor estimado aos 38,5 dias (Tabela 1). Nos cerrados de Rondônia, para pastagens de M. maximus cvs. Aruana, Tanzânia-1 e Vencedor, Costa et al. (2007) reportaram maiores rendimentos de forragem para períodos de descanso variando entre 28 e 35 dias. Para frequências de desfolhação maiores a velocidade de rebrota apresentou alta correlação com a preservação dos meristemas apicais, cuja preservação favorece a formação de tecidos fotossintetizantes por meio da expansão de novas folhas, pois a remoção de meristemas apicais implica em crescimento mais lento e originado a partir do desenvolvimento de gemas, notadamente de origem basal, para a produção de folhas (Difante et al., 2011, Cunha et al., 2012). Para Santos et al. (2003) o intervalo entre pastejos de M. maximus cv. Tanzânia-1 não deve ser estabelecido apenas com base na taxa de acúmulo de matéria seca, sendo necessário o conhecimento sobre as interações entre produção de hastes e eficiência de pastejo, consumo e qualidade de forragem. Os autores recomendam frequências de pastejo a cada 38 dias no período de outubro a abril, 28 dias na fase reprodutiva da gramínea (abril/maio) e com aproximadamente 48 dias entre maio e setembro. Baseados nesta premissa, Barbosa et al. (2007) constataram interação entre altura de resíduo pós-pastejo e frequência de desfolhação em pastagens de $M$. maximus cv. Tanzânia-1, sendo sugeridos períodos de descanso de 31 a 35 dias e, 24 a 27 dias, respectivamente para 25 e $50 \mathrm{~cm}$ de resíduo, os quais foram correlacionados com $90 \%$ de interceptação luminosa pelo dossel da pastagem.

Os teores de N, P, Ca Mg e K foram negativa e linearmente afetados pelos períodos de descanso (Tabela 1), evidenciando a diluição em suas concentrações com a redução da frequência de desfolhação da gramínea. Os teores registrados 
neste trabalho foram semelhantes ou superiores aos relatados por Costa et al. (2007) para pastagens de $M$. maximus cvs. Atlas, Centenário e Vencedor, submetidas a diferentes períodos de descanso, Para o P, Ca, Mg e K, os teores obtidos com frequências de desfolhação de até 35 dias, foram superiores ao nível crítico interno determinados por Costa et al. (2006) para $M$. maximus cv. Vencedor $(1,79 ; 3,45 ; 2,36$ e $18,76 \mathrm{~g}$ $\mathrm{kg}^{-1}$, respectivamente para $\mathrm{P}, \mathrm{Ca}, \mathrm{Mg} \mathrm{e} \mathrm{K}$ ). Contudo, Oliveira et al. (2009), para pastagens de $M$. maximus cv. Mombaça, relataram concentrações máximas de $\mathrm{N}, \mathrm{P}, \mathrm{K}, \mathrm{Ca}$ e $\mathrm{Mg}$, respectivamente aos 104, 102, 105, 68 e 78 dias de descanso.

A frequência de desfolhação afetou negativa $\mathrm{e}$ linearmente a TAF, enquanto que para a TEF, NFV, CMF e IAF os ajustes foram quadráticos e os máximos valores obtidos com frequências de desfolhação de 24,1; 32,3; 40,2 e 34,7 dias (Tabela 2). Nos cerrados de Rondônia, em pastagens de $M$. maximus cvs. Tobiatã e Vencedor, Costa et al. (2008) estimaram maiores NFV, CMF e IAF para períodos compreendidos entre 28 e 35 dias de rebrota, os quais foram recomendados como mais adequados para o manejo das gramíneas. Em pastagens de $M$. maximus cv. Tanzânia-1, o prolongamento do período de descanso afetou negativamente a estrutura de seu dossel, reduzindo a relação folha/colmo e a população de perfilhos, contudo, adaptações morfológicas e estruturais do dossel forrageiro permitiram satisfatória rebrota sob manejo em que a frequência entre desfolhações permite o aparecimento de 4,0 novas folhas perfilho ${ }^{-1}$, durante a época chuvosa. $\mathrm{O}$ período adequado de descanso em pastagens de capim-Tanzânia-1 não deve exceder 35 dias e o aparecimento de pelo menos 3,5 folhas totalmente expandidas por perfilho (Ferlin et al., 2006, Gomide et al., 2007).

O IAF representa a síntese das características morfogênicas e estruturais da gramínea e reflete $o$ balanço dos processos que determinam a oferta (fotossíntese) e a demanda (respiração, acúmulo de reservas, síntese e senescência de tecidos) de fotoassimilados, estabelecendo o ritmo de crescimento da pastagem (Nabinger \& Carvalho, 2009). Com o aumento da frequência de desfolhação, a percentagem de luz interceptada pelo dossel atinge seu ponto máximo, $\mathrm{IAF}_{\text {Teto }}$, onde para cada nova folha surgida na porção superior da planta ocorre a senescência de uma folha em sua porção inferior, estabilizando ou reduzindo a disponibilidade de biomassa verde como decorrência da redução do coeficiente de extinção luminosa (Cândido et al., 2005, Pereira, $\underline{2013}$ ).

Tabela 1. Produção de matéria seca verde (MSV - $\left.\mathrm{kg} \mathrm{ha}^{-1}\right)$, vigor de rebrota (VR - kg MSV/21 dias) e teores de nitrogênio (N), fósforo $(\mathrm{P})$, cálcio $(\mathrm{Ca})$, magnésio $(\mathrm{Mg})$ e potássio $(\mathrm{K})$ de Megathyrsus maximus cv. Tanzânia-1, em função da frequência de desfolhação.

\begin{tabular}{|c|c|c|c|c|c|c|}
\hline \multirow{2}{*}{ Variáveis } & \multicolumn{5}{|c|}{ Frequência de Desfolhação (dias) } & \multirow{2}{*}{ Equação de Regressão } \\
\hline & 14 & 21 & 28 & 35 & 42 & \\
\hline MSV & 1.107 & 1.645 & 2.879 & 3.321 & 2.899 & $\mathrm{Y}=3.319+284,41 \mathrm{X}-3,6914 \mathrm{X}^{2}\left(\mathrm{R}^{2}=0,93\right)$ \\
\hline $\mathrm{N}(\mathrm{g} / \mathrm{kg})$ & 29,55 & 27,88 & 27,21 & 26,35 & 24,91 & $Y=31,51-0,1544 X\left(r^{2}=0,93\right)$ \\
\hline $\mathrm{P}(\mathrm{g} / \mathrm{kg})$ & 2,17 & 2,02 & 1,91 & 1,84 & 1,73 & $Y=2,358-0,0151 X\left(r^{2}=0,95\right)$ \\
\hline $\mathrm{Ca}(\mathrm{g} / \mathrm{kg})$ & 4,81 & 4,17 & 3,56 & 3,22 & 3,08 & $Y=5,532-0,06037 X\left(r^{2}=0,94\right)$ \\
\hline $\mathrm{Mg}(\mathrm{g} / \mathrm{kg})$ & 3,11 & 2,89 & 2,76 & 2,59 & 2,31 & $\mathrm{Y}=3,492-0,02712 \mathrm{X}\left(\mathrm{r}^{2}=0,95\right)$ \\
\hline $\mathrm{K}(\mathrm{g} / \mathrm{kg})$ & 21,07 & 18,89 & 17,56 & 17,02 & 16,55 & $Y=22,582-0,1559 X\left(r^{2}=0,89\right)$ \\
\hline
\end{tabular}

O CMF é a característica plástica mais responsiva à intensidade e frequência de desfolhação e considerada como a principal estratégia morfológica de escape das plantas ao pastejo (Pedreira et al., 2009, Lemaire et al., 2011). As gramíneas sob desfolhações frequentes geralmente apresentam abundante perfilhamento, hábito de crescimento mais prostrado e elevado ritmo de expansão foliar, possibilitando maior interceptação de luz e rebrotas mais vigorosas
(Nabinger \& Pontes, 2002, Costa et al. 2014). As TAF, TEF e CMF obtidas neste trabalho, independentemente das frequências de desfolhação, foram superiores às reportadas por Macedo et al. (2010) avaliando M. maximus cv. Mombaça que constataram valores médios de 0,011 e 0,010 folhas $^{-1}$ perfilho $^{-1}$ dia; 5,38 e $5,31 \mathrm{~cm}$ dia $^{-1}$ perfilho $^{-1}$ e 40,5 e $33,2 \mathrm{~cm}$ para o CMF, respectivamente para períodos de descanso de 36 e 48 dias. O NFV torna-se constante a partir do 
momento em que a TSF se iguala a TAF e constitui critério objetivo e prático para definição dos períodos de descanso na lotação rotativa e a intensidade de pastejo na lotação contínua (Santos et al., 2004, Pereira, 2013).

Para pastagens de M. maximus cv. Vencedor, Costa et al. (2008) recomendam o início do pastejo quando os perfilhos apresentarem, em média, três a quatro folhas vivas, de modo a potencializar a produtividade e a qualidade da forragem em oferta. A TEF apresenta alta correlação com a produção de MSV e tem sido utilizada como critério para a seleção de gramíneas em trabalhos de melhoramento genético (Nabinger \& Carvalho, 2009, Pereira, 2013). A TEF está diretamente correlacionada com o CMF, pois folhas menores estão normalmente associadas a maiores valores de TAF. Neste trabalho a correlação entre TEF e rendimento de MSV foi positiva e significativa ( $\mathrm{r}$ $=0,852 ; \mathrm{P}<0,05)$; enquanto que com a TAF a correlação foi negativa e não significativa $(\mathrm{r}=$ $0,572$; $\mathrm{P}>0,05)$. A TEF explicou em $72,6 \%$ os incrementos nos rendimentos de MSV, em função da frequência de desfolhação. A TAF é a característica morfogênica mais relevante, pois afeta diretamente as três características estruturais do relvado: tamanho da folha, densidade de perfilhos e número de folhas perfilho ${ }^{-1}$ (Pedreira et al., 2009, Santos et al., 2012). Para Difante et al. (2011) a TAF e a TEF apresentam, geralmente, correlação negativa, indicando que quanto maior a TAF, menor será o tempo disponível para o alongamento foliar (Nabinger \& Carvalho, 2009).

Tabela 2. Número de folhas vivas (NFV), taxa de aparecimento de folhas (TAF - folhas dia ${ }^{-1}$ perfilho-1 ${ }^{-1}$, taxa de expansão foliar (TEF - cm dia ${ }^{-1}$ perfilho-1), comprimento médio de folhas (CMF - cm), densidade populacional de perfilhos $\mathrm{m}^{-2}$ (DPP), índice de área foliar (IAF) e taxa de senescência foliar (TSF - $\mathrm{cm} \mathrm{dia}^{-1}$ perfilho ${ }^{-1}$ ) de Megathyrsus maximus cv. Tanzânia-1, em função da frequência de desfolhação.

\begin{tabular}{|c|c|c|c|c|c|c|}
\hline \multirow{2}{*}{ Variáveis } & \multicolumn{5}{|c|}{ Frequência de Desfolhação (dias) } & \multirow{2}{*}{ Equação de Regressão } \\
\hline & 14 & 21 & 28 & 35 & 42 & \\
\hline NFV & 2,97 & 3,57 & 3,93 & 4,11 & 3,71 & $\mathrm{Y}=0,672+0,2068 \mathrm{X}-0,0032 \mathrm{X}^{2}\left(\mathrm{R}^{2}=0,91\right)$ \\
\hline TAF & 0,212 & 0,170 & 0,143 & 0,117 & 0,088 & $Y=0,2657-0,00433 X\left(r^{2}=0,94\right)$ \\
\hline TEF & 3,36 & 3,05 & 3,56 & 3,44 & 2,35 & $Y=1,609+0,0156 X-0,0032 X^{2}\left(r^{2}=0,87\right)$ \\
\hline $\mathrm{CMF}$ & 15,85 & 17,92 & 25,37 & 29,33 & 26,55 & $\mathrm{Y}=3,312+1,5454 \mathrm{X}-0,0192 \mathrm{X}^{2}\left(\mathrm{R}^{2}=0,93\right)$ \\
\hline DPP & 457 & 401 & 387 & 322 & 299 & $Y=531,2-5,6429 X\left(r^{2}=0,92\right)$ \\
\hline IAF & 1,35 & 2,17 & 2,98 & 3,54 & 2,87 & $Y=2,412+0,3267 X-0,00472 X^{2}\left(R^{2}=0,90\right)$ \\
\hline TSF & 0,121 & 0,139 & 0,152 & 0,159 & 0,172 & $\mathrm{Y}=0,0998+0,00179 \mathrm{X}\left(\mathrm{r}^{2}=0,95\right)$ \\
\hline
\end{tabular}

A DPP foi linear e inversamente proporcional à frequência de desfolhação (Tabela 2). O perfilhamento constitui característica estrutural fortemente influenciada por fatores nutricionais, ambientais e de manejo, que definem as características morfogênicas que condicionam a resposta morfogênica das plantas forrageiras aos sistemas de manejo (Cecato et al., 2000; Garcez Neto et al., 2002). A produção de novos perfilhos é um processo contínuo e acelerado pela desfolhação da planta em decorrência da melhoria do ambiente luminoso na base do dossel (maior razão da radiação vermelha:vermelha distante), sendo intermediada por dois fatores principais: o suprimento de energia para a fotossíntese e o número e atividade de pontos de crescimento (Gastal \& Lemaire, 2002; Nabinger \& Carvalho, 2009). Em pastagens de M. maximus cv. Centenário, Costa et al. (2008) reportaram maior
DPP para desfolhações a $20 \mathrm{~cm}\left(678\right.$ perfilhos m${ }^{-}$ $\left.{ }^{2}\right)$, comparativamente a $30\left(538\right.$ perfilhos $\left.\mathrm{m}^{-2}\right)$ ou $40 \mathrm{~cm}$ acima do solo (399 perfilhos $\mathrm{m}^{-2}$ ). Corsi (1984) observou intensa concentração na emissão de perfilhos nos primeiros oito dias após o corte em ecotipos de $M$. maximus, enquanto que Barbosa et al. (2002), avaliando quatro cultivares de $M$. maximus, constataram aparecimento linear de perfilhos, basilares e aéreos, até 21 dias após o corte. A maior quantidade de perfilhos basilares, notadamente nas primeiras semanas, foi consequência da maior intensidade luminosa sobre as gemas basilares, a qual estimulou o perfilhamento (Pereira, 2013).

A TSF foi inversamente proporcional à frequência de desfolhação, evidenciando os efeitos negativos de menores frequências de desfolhação sobre a qualidade da forragem (Tabela 2). A senescência foliar decorre da 
competição por metabólitos e nutrientes entre folhas velhas e em crescimento, o que reduz a disponibilidade de forragem de boa qualidade (Santos et al., 2004, Lemaire et al., 2011). Os valores estimados foram inferiores aos reportados por Costa et al. (2007) para pastagens de $M$. maximus cv. Tobiatã $\left(0,197 ; 0,204\right.$ e $0,245 \mathrm{~cm} \mathrm{dia}^{-}$ ${ }^{1}$ perfilho $^{-1}$, respectivamente para frequências de desfolhação de 21, 35 e 42 dias). A senescência caracteriza a última fase de desenvolvimento da folha, após sua completa expansão e acentuada com o incremento da área foliar, em função do sombreamento das folhas inseridas na porção inferior e do baixo suprimento de radiação fotossinteticamente ativa, além de forte competição por luz, nutrientes e água entre os diversos estratos da planta (Nabinger \& Pontes, 2002). O perfilho ao atingir determinado NFV estabelece o equilíbrio entre TAF e senescência das folhas que ultrapassaram seu período de duração de vida, de modo que o surgimento de nova folha implica na senescência da folha que a precedeu, mantendo o NFV relativamente constante (Lemaire et al., 2011; Pereira, 2013). A senescência reduz a qualidade da forragem, contudo representa importante processo fisiológico no fluxo de tecidos da gramínea, pois cerca de 35; 68; 86 e 42\% do nitrogênio, fósforo, potássio e magnésio, respectivamente, podem ser reciclados das folhas senescentes e utilizados para a produção de novos tecidos foliares (Sarmiento et al., 2006, Costa et al., 2013).

\section{Conclusões}

A redução na frequência de desfolhação favorece os rendimentos de forragem e o vigor de rebrota, contudo reduz os teores de $\mathrm{N}, \mathrm{P}, \mathrm{Ca}, \mathrm{Mg}$ e K. A taxa de aparecimento de folhas e a densidade populacional de perfilhos são inversamente proporcionais as frequências de desfolhação, ocorrendo o inverso quanto ao comprimento médio de folhas, índice de área foliar e taxa de senescência foliar. A frequência de desfolhação mais adequada para pastagens de $M$. maximus cv. Tanzânia-1, visando conciliar produção, vigor de rebrota e qualidade da forragem, situa-se entre 35 e 40 dias.

\section{Referências Bibliográficas}

Barbosa, R. A., Nascimento Júnior, D., Euclides, V. P. B., Regazzi, A. J. \& Fonseca, D. M. 2002. Características morfogênicas e acúmulo de forragem do capim-tanzânia. Revista Brasileira de Zootecnia, 31, 583-593.
Barbosa, R.A., Nascimento Júnior, D., Euclides, V.P.B., Silva, S.C., Zimmer, A.H. \& Torres Júnior, R.A.A. 2007. Capim-tanzânia submetido a combinações entre intensidade e frequiência de pastejo. Pesquisa Agropecuária Brasileira, 42, 329-340.

Cândido, M. J. D., Alexandrino, E. \& Gomide, J. A. 2005. Duração do período de descanso e crescimento do dossel de Panicum maximum cv. Mombaça sob lotação intermitente. Revista Brasileira de Zootecnia, 34, 398-405.

Cecato, U., Machado, A. O., Martins, E. N. \& Santos, G. T. 2000. Avaliação da produção e de algumas características da rebrota de cultivares e acessos de Panicum maximum Jacq. sob duas alturas de corte. Revista Brasileira de Zootecnia, 9, 660-668.

Corsi, M. 1984. Effects of nitrogen rates and harvesting intervals on dry matter productivity, tillering and quality of tropical grass Panicum maximun Jacq. 125p. Thesis (Ph.D.) Ohio State University, Wooster, 1984.

Costa, C. A. A., Andrade, A. C., Magalhães, J. A., Mehl, H. U., Rodrigues, B. H. N., Silva, E. M. \& Costa, N. L. 2014. Características agronômicas dos capins Digitaria sp. e Cynodon dactylon cv. Tifton-85 sob diferentes alturas de resíduo. PubVet, 7, 1509.

Costa, N.L., Paulino, V.T., Magalhães, J.A., Townsend, C.R. \& Pereira, R.G.A. 2008. Morfogênese de gramíneas forrageiras na Amazônia Ocidental. Pubvet, 2, 1-24.

Costa, N.L., Townsend, C.R., Magalhães, J.A. \& Pereira, R.G.A. 2006. Formação e manejo de pastagens na Amazônia do Brasil. Revista Electrónica de Veterinária, 7, 1-18.

Costa, N. L., Magalhães, J. A., Pereira, R. G. A., Townsend, C. R. \& Oliveira, J. R. C. 2007. Considerações sobre o manejo de pastagens na Amazônia Ocidental. Revista do Conselho Federal de Medicina Veterinária, 40, 37-56.

Costa, N. L., Moraes, A., Monteiro, A. L. G., Motta, A. C. V., Oliveira, R. A. \& Rodrigues, A. N. A. 2013. Forage productivity and morphogenesis of Axonopus aureus under different nitrogen fertilization rates. Revista Brasileira de Zootecnia, 42, 541-548.

Cunha, F. F., Mota Ramos, M., Brasileiro, A. C. A., Alves , O., Rubens, Cóser, A. C. \& Silva, A. R. A. 2012. Produtividade da Brachiaria brizantha cv. Xaraés em diferentes manejos e 
doses de adubação, períodos de descanso e épocas do ano. Idesia (Arica), 30, 75-82.

Difante, G. S., Nascimento Júnior, D., Silva, S. C., Euclides, V. P. B. \& Montagner, D. B. 2011. Características morfogênicas e estruturais do capim-marandu submetido a combinações de alturas e intervalos de corte. Revista Brasileira de Zootecnia, 40, 955-963.

Ferlin, M. B., Euclides, V. P. B., Lempp, B. \& Cubas, C. C. 2006. Morfogênese e dinâmica de perfilhamento de Panicum maximum Jacq. cv. Tanzânia-1 sob pastejo. Ciência $e$ Agrotecnologia, 30, 344-352.

Garcez Neto, A. F., Nascimento Júnior, D., Regazzia, A. J. \& Gobbi, K. F. 2002. Respostas morfogênicas e estruturais de Panicum maximum cv. Mombaça sob diferentes níveis de adubação nitrogenada e alturas de corte. Revista Brasileira de Zootecnia, 31, 18901900

Gastal, F. \& Lemaire, G. 2002. N uptake and distribution in crops: an agronomical and ecophysiological perspective. Journal of Experimental Botany, 53, 789-799.

Gomide, C. A. M., Gomide, J. A. \& Alexandrino, E. 2007. Características estruturais e produção de forragem em pastos de capim-mombaça submetidos a períodos de descanso. Pesquisa Agropecuária Brasileira, 42, 1487-1494.

Lemaire, G., Hodgson, J. \& Chabbi, A. 2011. Grassland productivity and ecosystem services. Cabi, Wallingford. 287p.

Macedo, C. H. O., Alexandrino, E., Jakelaitis, A., Vaz, R. G. \& Vendrusculo, J. 2010. Características agronômicas, morfogênicas e estruturais do capim" Panicum maximum" cv. Mombaça sob desfolhação intermitente. Revista Brasileira de Saúde e Produção Animal, 11, 941-952.

Nabinger, C. \& Carvalho, P. C. F. 2009. Ecofisiología de sistemas pastoriles: aplicaciones para su sustentabilidad. Agrociencia, 13, 18-27.

Nabinger, C. \& Pontes, L. S. 2002. Manejo da desfolha. Simpósio sobre manejo da pastagem. FEALQ, Jaboticabal. p.133-158.
Oliveira, P. S. R., Castagnara, D. D., Júnior Gonçalves, A. C., Mesquita, E. E. \& Neres, M. A. 2009. Teores de macrominerais em Panicum maximum cvs. Massai e Mombaça. Synergismus Scyentifica, 4, 7-10.

Pedreira, B., Pedreira, C. G. S. \& Silva, S. C. 2009. Acúmulo de forragem durante a rebrotação de capim-xaraés submetido a três estratégias de desfolhação. Revista Brasileira de Zootecnia, 38, 618-625.

Pereira, V.V. 2013. A importância das características morfogênicas sobre o manejo de pastagens tropicais. Revista Agronegócios e Meio Ambiente, 6, 289-309.

Santos, M. R., Fonseca, D. M., Gomes, V. M., Silva, S. P., Silva, G. P. \& Reis, M. 2012. Correlações entre características morfogênicas e estruturais em pastos de capim-braquiária. Ciência Animal Brasileira, 13, 49-56.

Santos, P. M., Balsalobre, M. A. A. \& Corsi, M. 2004. Características morfogenéticas e taxa de acúmulo de forragem do capim-mombaça submetido a três intervalos de pastejo. Revista Brasileira de Zootecnia, 33, 843-851.

Santos, P. M., Balsalobre, M.A.A. \& Corsi, M. 2003. Morphogenetic characteristics and management of Tanzania grass. Pesquisa Agropecuária Brasileira, 38, 991-997.

Sarmiento, G., Silva, M. P., Naranjo, M. E. \& Pinillos, M. 2006. Nitrogen and phosphorus as limiting factors for growth and primary production in a flooded savanna in the Venezuelan Llanos. Journal of Tropical Ecology, 22, 203-212.

\section{Article History:}

Received 5 January 2018

Accepted 16 February 2018

Available on line 14 April 2018

License information: This is an open-access article distributed under the terms of the Creative Commons Attribution License 4.0, which permits unrestricted use, distribution, and reproduction in any medium, provided the original work is properly cited. 